\title{
Silagem de híbridos de sorgo associado a percentagens de concentrado no desempenho de novilhos
}

\author{
[Effect of sorghum hybrids silage associated to percentage of concentrate levels on the \\ performance of steers] \\ J. Restle ${ }^{1}$, R.L. Missio ${ }^{2}$, P.L.P. Resende ${ }^{3}$, N.L.Q. Silva ${ }^{4}$, F.N. Vaz ${ }^{4}$ I.L. Brondani ${ }^{4}$, \\ D.C. Alves Filho ${ }^{4}$, F. Kuss 5 \\ ${ }^{1}$ Universidade Federal do Tocantins (UFT) - Araguaína, TO \\ ${ }^{2}$ Pós-doutorando - UFT - Araguaína, TO \\ ${ }^{3}$ Universidade Federal de Goiânia - Goiânia, GO \\ ${ }^{4}$ Universidade Federal de Santa Maria - Santa Maria, RS \\ ${ }^{5}$ Instituto Federal do Paraná - campus Dois Vizinhos, PR
}

\begin{abstract}
RESUMO
Avaliou-se o desempenho de novilhos alimentados em confinamento com silagem de híbridos de sorgo forrageiro e de duplo propósito associado a níveis de concentrado. Foram utilizados 60 novilhos, com idade inicial de 20 meses e peso corporal inicial de $322,45 \pm 5,58 \mathrm{~kg}$, distribuídos inteiramente ao acaso, nos seguintes tratamentos: três porcentagens de concentrado e dois tipos de silagem de sorgo. Foi verificada interação tipo de silagem versus porcentagem concentrado para consumo de matéria seca e ganho de peso médio diário, os quais aumentaram com o aumento de concentrado na dieta com silagem de sorgo forrageiro, mas não com silagem de sorgo duplo propósito. Os novilhos alimentados com silagem de sorgo forrageiro consumiram menos energia digestível $\left(27,89 \mathrm{Mcal} / \mathrm{dia}^{-1}\right)$ que aqueles alimentados com silagem de sorgo duplo propósito $\left(31,42 \mathrm{Mcal} / \mathrm{dia}^{-1}\right)$. A conversão alimentar média não foi alterada pelo tipo de silagem utilizada. $\mathrm{O}$ aumento da porcentagem de concentrado em dietas com volumoso de menor qualidade aumenta a eficiência biológica de bovinos confinados.
\end{abstract}

Palavras-chave: bovino de corte, ganho de peso, consumo, sorgo forrageiro, sorgo duplo propósito

\begin{abstract}
The feedlot performance of steers fed forage sorghum and double purpose hybrids silage associated with concentrate levels was evaluated. A total of 60 steers, initial age 20 months and body weight of $322.45 \pm 5.58 \mathrm{~kg}$, were randomized in the treatments: three concentrate levels and two types of sorghum silage. The interaction of sorghum silage versus concentrate levels was observed for dry matter intake and average daily weight gain, which increased with the increase concentrate level in diets with forage sorghum silage, but was not altered in diets with double purpose silage. Steers fed forage sorghum silage presented a lower average intake of digestible energy $\left(27.89 \mathrm{Mcal}_{\text {/day }}{ }^{-1}\right)$ than those fed double purpose sorghum silage $\left(31.42 \mathrm{Mcal} / \mathrm{day}^{-1}\right)$. The average feed conversion was not affected by the type of silage used. The increase of concentrate level in diets with lower forage quality increases the biological efficiency of feedlot beef cattle.
\end{abstract}

Keywords: beef cattle, double purpose sorghum, forage sorghum, intake, and weight gain

Recebido em 19 de abril de 2011

Aceito em 30 de maio de 2012

E-mail: jorestle@terra.com.br 


\section{INTRODUÇÃO}

Embora os índices apontem baixa eficiência produtiva, o Brasil tem elevado a produtividade do rebanho bovino com aptidão para corte ao longo do tempo, aumentando em $10 \%$ a taxa de abate na última década (Anuário..., 2010). Além disso, alguns indicadores, como o aumento do número de animais em confinamento (Anuário..., 2010) em épocas críticas do ano, têm mostrado investimentos para contornar os problemas da produção sazonal de forragens e aumentar a produção de carne brasileira, apesar de essa prática ser considerada de alto custo.

Os custos do confinamento têm sido o maior entrave para sua utilização, devido à valorização dos grãos usados na alimentação animal. Apesar disso, a utilização de dietas à base de grãos tem aumentado nos últimos anos no Brasil, principalmente em confinamentos industriais. Tal fato pode vir a gerar prejuízos financeiros aos pecuaristas, uma vez que ocorre elevação do preço internacional do milho, motivado pela produção de bicombustível. Atualmente os EUA destinam $40 \%$ do milho produzido para produção de bicombustíveis (Wilkinson, 2010), alterando o preço internacional das commodities.

Para obter lucratividade na terminação de bovinos em confinamento, é recomendada a redução dos custos com alimentação, pois esse componente é o mais expressivo, quando desconsiderado o valor de compra do animal, superando $70 \%$ do custo de produção, sendo o concentrado o componente mais oneroso da dieta
(Pacheco et al., 2006; Restle et al., 2007). Como alternativa, tem sido proposta (Restle et al., 2007; Chizzotti et al., 2010) a utilização de silagem com maior participação de grãos, com o intuito de substituir parte dos nutrientes fornecidos pelo concentrado. Sendo assim, o presente estudo teve o objetivo de avaliar o desempenho de novilhos alimentados em confinamento com silagem de dois híbridos de sorgo, forrageiro e de dupla aptidão, associado a três porcentagens de concentrado na dieta.

\section{MATERIAL E MÉTODOS}

Foram utilizados 60 novilhos, mestiços CharolêsNelore, contemporâneos, com idade inicial de 20 meses e peso corporal inicial de $322,45 \pm 5,58 \mathrm{~kg}$, distribuídos inteiramente ao acaso em seis tratamentos de três porcentagens de concentrado e dois tipos de silagem de sorgo. Os animais, submetidos a 14 dias de adaptação às instalações e às dietas, foram alimentados à vontade, em duas refeições diárias, as oito e às 17 horas, durante o período experimental de 140 dias. As dietas, isonitrogenadas, foram balanceadas para ganho de peso de $1,2 \mathrm{~kg} / \mathrm{dia}^{-1}$, considerando-se o consumo de $2,4 \%$ do peso corporal segundo National Research Council-NRC, Nutrients..., (1996). O consumo foi registrado diariamente por meio da pesagem dos alimentos e das sobras do dia anterior. Os volumosos utilizados foram silagem de sorgo forrageiro (AG2002 ${ }^{\circledR}$ ) e sorgo duplo propósito (AG2005 ${ }^{@}$ ). O concentrado foi constituído de grão de sorgo triturado, farelo de soja, ureia, sulfato de amônia, calcário calcítico e sal comum (Tab. 1).

Tabela 1. Composição do concentrado de acordo com a porcentagem de concentrado na dieta para novilhos de corte

\begin{tabular}{lccc}
\hline \multirow{2}{*}{ Ingrediente $(\%$ da MS) } & \multicolumn{3}{c}{ Concentrado (\%) } \\
\cline { 2 - 4 } & 25 & 35 & 45 \\
\hline Sorgo grão triturado & 83,40 & 89,00 & 91,60 \\
Farelo de soja & 8,90 & 4,30 & 2,40 \\
Uréia & 3,30 & 2,40 & 1,80 \\
Sulfato de amônia & 0,40 & 0,30 & 0,20 \\
Calcário calcítico & 3,00 & 3,00 & 3,00 \\
Sal comum & 1,00 & 1,00 & 1,00 \\
\hline
\end{tabular}

MS = matéria seca.

Os animais foram pesados no início e no final de cada período (28 dias), após jejum de sólidos e líquidos de 14 horas, sendo abatidos à medida que atingiam o peso predeterminado $(420 \mathrm{~kg})$. Durante o período de confinamento, foram coletadas amostras dos alimentos e sobras semanalmente. As amostras foram pré-secas em estufa de ar forçado a $55^{\circ} \mathrm{C}$, por 72 horas, processadas em moinho tipo Willey com peneira com crivos de $1 \mathrm{~mm}$, utilizadas para 
determinação da composição dos alimentos (Tab. 2). Nas amostras foram determinados os teores de matéria seca, cinzas e proteína bruta segundo AOAC Official... (1995). Os teores de fibra em detergente neutro e fibra em detergente ácido foram determinados de acordo com Robertson e Van Soest (1981). A digestibilidade in vitro da matéria seca foi determinada pelo método de Tilley e Terry (1963). A energia digestível (ED) e o teor de nutrientes digestíveis totais (NDT) foram determinados segundo equações do ARC Nutrient... (1980), em que: ED = MOD (matéria orgânica digestível) $x 0,19 / 4,184$ e NDT $=($ ED x 100)/4,4.

Tabela 2. Composição dos ingredientes das dietas fornecidas a novilhos de corte

\begin{tabular}{lcccc}
\hline Item (\% da matéria seca) & Silagem SF & Silagem DP & Sorgo grão & Farelo soja \\
\hline Matéria seca, \% & 30,20 & 33,40 & 88,00 & 88,00 \\
Matéria mineral & 3,10 & 4,10 & 0,80 & 5,30 \\
Proteína bruta & 6,40 & 7,20 & 9,20 & 44,00 \\
Fibra em detergente neutro & 57,10 & 52,50 & 16,1 & 14,10 \\
Fibra em detergente ácido & 33,10 & 28,90 & 6,40 & 10,00 \\
Digestibilidade in vitro da MS & 57,80 & 70,20 & 81,7 & 84,50 \\
Digestibilidade in vitro da MO & 59,00 & 70,00 & 84,20 & 80,80 \\
Nutrientes digestíveis totais & 59,00 & 70,00 & 84,2 & 80,80 \\
Energia digestível & 2,50 & 3,00 & 3,70 & 3,50 \\
\hline
\end{tabular}

$\mathrm{SF}=$ sorgo forrageiro; $\mathrm{DP}=$ sorgo duplo propósito.

O delineamento experimental foi o inteiramente casualizado, com os tratamentos organizados em esquema fatorial $3 \times 2$, três níveis de concentrado e dois tipos de silagem de sorgo. Os dados foram submetidos à análise de variância e ao teste de Tukey para a comparação entre silagens e à análise de regressão polinomial para o efeito do concentrado na dieta, considerando-se $5 \%$ como o nível crítico de significância.

\section{RESULTADOS E DISCUSSÃO}

Foram verificadas interações significativas entre o tipo de híbrido de sorgo utilizado para silagem e a porcentagem de concentrado da dieta para as variáveis estudadas. Nesse sentido, verificou-se que o consumo de matéria seca, nas formas como foi expresso, aumentou linearmente com a elevação da fração concentrada nas dietas com silagem de sorgo forrageiro (SF), não sendo alterado nas dietas com silagem de sorgo duplo propósito (SDP) (Tab. 3).

O aumento da ingestão nas dietas com SF pode estar associado à elevação do teor energético e à diminuição do teor de fibra das dietas com o aumento da porcentagem de concentrado. De outra forma, com os resultados obtidos infere-se que a associação entre a silagem de SF e os menores níveis de concentrado testados limitou o consumo de matéria seca, em virtude da limitação física do retículo-rúmen, fato também atribuído por Neumann et al. (2001), os quais verificaram menor consumo de alimento em novilhos alimentados com dietas com silagem de SF (AGX-213 e AG-2002) em relação àqueles alimentados com silagem de SDP (AGX-217 e AG-2005E). Araújo et al. (1998) e Missio et al. (2009) verificaram que o consumo de matéria seca apresentou variação quadrática devido ao aumento da porcentagem de concentrado na dieta, com máximo consumo quando os animais foram alimentados com $67 \%$ de concentrado, aproximadamente. De forma geral, os resultados destes autores revelaram que o consumo de matéria seca tende a aumentar com a inclusão de concentrado na dieta, enquanto a proporção de volumoso (de média a alta qualidade) permaneceu maior que a de concentrado. Todavia, à medida que a fração de concentrado foi superando a de volumoso, o consumo tendeu a se aproximar do ponto de inflexão da curva, a partir do qual diminuiu, em virtude do aumento no consumo de energia digestível e atendimento da demanda fisiológica de energia para o animal. No entanto, tais variações do consumo em razão da elevação do concentrado na dieta não têm sido unanimidade entre as pesquisas (Ítavo et al., 2002; Silva et al., 2005). 
Tabela 3. Consumo das frações alimentares em função da silagem e da porcentagem de concentrado na dieta de novilhos de corte

\begin{tabular}{|c|c|c|c|c|c|c|c|}
\hline \multirow{2}{*}{ Híbrido } & \multicolumn{3}{|c|}{ Concentrado (\%) } & \multirow{2}{*}{ Média } & \multirow{2}{*}{ Regressão } & \multirow{2}{*}{$\mathrm{R}^{2}$} & \multirow{2}{*}{$\mathrm{CV}$} \\
\hline & 25 & 35 & 45 & & & & \\
\hline \multicolumn{8}{|c|}{ Consumo de matéria seca, $\mathrm{kg} / \mathrm{dia}^{-1}$} \\
\hline $\mathrm{SF}$ & $9,09 \mathrm{~A}$ & $9,32 \mathrm{~A}$ & $10,39 \mathrm{~A}$ & $9,60 \mathrm{~A}$ & $\mathrm{Y}=7,443+0,0614 \mathrm{X}$ & 0,65 & \multirow{2}{*}{3,81} \\
\hline SDP & $9,52 \mathrm{~A}$ & $9,97 \mathrm{~A}$ & $9,82 \mathrm{~A}$ & $9,77 \mathrm{~A}$ & $Y=9,76$ & -- & \\
\hline \multicolumn{8}{|c|}{ Consumo de matéria seca, $\%$ do peso corporal } \\
\hline $\mathrm{SF}$ & $2,44 \mathrm{~A}$ & $2,52 \mathrm{~A}$ & $2,76 \mathrm{~A}$ & $2,44 \mathrm{~A}$ & $\mathrm{Y}=2,002+0,016 \mathrm{X}$ & 0,68 & \multirow{2}{*}{4,11} \\
\hline SDP & $2,57 \mathrm{~A}$ & $2,65 \mathrm{~A}$ & $2,61 \mathrm{~A}$ & $2,57 \mathrm{~A}$ & $\mathrm{Y}=2,59$ & -- & \\
\hline \multicolumn{8}{|c|}{ Consumo de fibra em detergente neutro, $\mathrm{kg} / \mathrm{dia}^{-{ }^{-}}$} \\
\hline $\mathrm{SF}$ & $4,24 \mathrm{~A}$ & $3,95 \mathrm{~A}$ & $3,98 \mathrm{~A}$ & $4,10 \mathrm{~A}$ & $\mathrm{Y}=4,057$ & -- & \multirow{2}{*}{3,75} \\
\hline SDP & $4,11 \mathrm{~A}$ & $3,92 \mathrm{~A}$ & $3,51 \mathrm{~B}$ & $3,85 \mathrm{~A}$ & $\mathrm{Y}=4,888-0,0298 \mathrm{X}$ & 0,81 & \\
\hline \multicolumn{8}{|c|}{ Consumo de fibra em detergente neutro, $\%$ do peso corporal } \\
\hline $\mathrm{SF}$ & $1,13 \mathrm{~A}$ & $1,07 \mathrm{~A}$ & $1,06 \mathrm{~A}$ & $1,08 \mathrm{~A}$ & $\mathrm{Y}=1,083$ & -- & \multirow{2}{*}{4,11} \\
\hline SDP & $1,10 \mathrm{~A}$ & $1,04 \mathrm{~A}$ & $0,93 \mathrm{~B}$ & $1,02 \mathrm{~A}$ & $Y=1,315-0,0083 X$ & 0,80 & \\
\hline \multicolumn{8}{|c|}{ Consumo de energia digestível, Mcal/dia } \\
\hline $\mathrm{SF}$ & $25,45 \mathrm{~B}$ & $26,98 \mathrm{~B}$ & $31,25 \mathrm{~A}$ & $27,89 \mathrm{~B}$ & $\mathrm{Y}=17,782+0,289 \mathrm{X}$ & 0,87 & \multirow{2}{*}{3,59} \\
\hline SDP & $30,11 \mathrm{~A}$ & $32,00 \mathrm{~A}$ & $32,14 \mathrm{~A}$ & $31,42 \mathrm{~A}$ & $Y=27,864$ & -- & \\
\hline \multicolumn{8}{|c|}{ Consumo de energia digestível, \% do peso corporal } \\
\hline $\mathrm{SF}$ & $6,77 \mathrm{~B}$ & $7,27 \mathrm{~B}$ & $8,30 \mathrm{~A}$ & $7,45 \mathrm{~B}$ & $\mathrm{Y}=4,78+0,0672 \mathrm{X}$ & 0,88 & \multirow{2}{*}{3,95} \\
\hline SDP & $8,06 \mathrm{~A}$ & $8,50 \mathrm{~A}$ & $8,53 \mathrm{~A}$ & $8,36 \mathrm{~A}$ & $\mathrm{Y}=7,528$ & -- & \\
\hline
\end{tabular}

SF e SDP = sorgo forrageiro e sorgo duplo propósito, respectivamente. Médias seguidas de letras maiúsculas distintas na linha diferem entre si pelo teste Tukey a $5 \%$ de probabilidade; $\mathrm{X}=$ porcentagem de concentrado na dieta.

A não variação do consumo de matéria seca com a elevação da porcentagem de concentrado nas dietas com SDP pode ser atribuída à maior densidade energética das dietas, já que, em virtude da maior produção de grãos, este híbrido contribuiu com $11 \%$ a mais de nutrientes digestíveis totais na silagem em relação ao SF. Tal fato possivelmente favoreceu o consumo de energia digestível em ambos os níveis de concentrado, não alterando o consumo devido ao controle quimiostático, conforme proposto por Van Soest (1994). Entretanto os resultados de consumo apresentados na literatura são controversos, apesar de serem verificadas tendências comuns em alguns trabalhos de pesquisa. Em geral, quando o volumoso da dieta apresenta elevado valor nutritivo (silagem de milho, sorgo ou fenos de boa qualidade), o consumo de matéria seca tende a manter-se constante (Ítavo et al., 2002; Véras et al., 2008) ou a diminuir (Bürger et al., 2000; Restle et al., 2002) com o aumento do concentrado da dieta, devido ao consumo de energia. Por outro lado, quando o volumoso é de baixa qualidade (silagens de capim, bagaço de cana-de-açúcar ou fenos de má qualidade), o consumo pode ser elevado linearmente em razão do aumento do nível de concentrado (Tibo et al., 2000; Silva et al., 2005; Vieira et al., 2010), resultado da demanda energética pelo animal e da maior capacidade de ingestão devido à redução do teor de fibra da dieta.

O consumo de fibra em detergente neutro não foi alterado $(\mathrm{P}>0,05)$ pela elevação da porcentagem de concentrado na dieta com SF, enquanto nas dietas com SDP, o consumo desta fração alimentar foi diminuído pela elevação do concentrado na dieta (Tab. 3). Tal variação, no caso das dietas com SDP, está relacionada à diminuição do teor de fibra em detergente neutro em virtude da maior participação da fração concentrada na dieta. Já a não variação do consumo de fibra em detergente neutro, nas dietas com SF, é resultado da compensação existente entre consumo de matéria seca e teor de fibra em detergente neutro. Nesse sentido, no menor nível de concentrado, o consumo foi menor, mas com maior teor de fibra, enquanto o maior consumo de matéria seca nas maiores porcentagens de concentrado ocorreu com menor teor de fibra, devido à maior participação do concentrado na dieta. Mertens (1994) afirmou que o consumo é limitado pelo efeito físico de enchimento quando o consumo de fibra em detergente neutro é de 11 a $13 \mathrm{~g} \mathrm{~kg}^{-1}$ de peso 
corporal. Neste experimento, o consumo de fibra em detergente neutro, nas dietas com silagem de $\mathrm{SF}$, foi de 11,$28 ; 10,64$ e $10,57 \mathrm{~g} \mathrm{~kg}^{-1}$ de peso corporal para os animais que receberam $25,35 \mathrm{e}$ 45 de concentrado, respectivamente, e nas dietas com SDP foi de 10,98; 10,43 e 9,32 $\mathrm{g} \mathrm{kg}^{-1}$ de peso corporal para a mesma porcentagem de concentrado, respectivamente.

O consumo de energia digestível, em $\mathrm{Mcal} / \mathrm{dia}^{-1}$ e $\%$ do peso corporal, aumentou linearmente em virtude da elevação da porcentagem de concentrado nas dietas com SF, não sendo alterado nas dietas com SDP (Tab. 3). O aumento no consumo de energia digestível para os animais alimentados com silagem de SF é semelhante ao verificado por Missio et al. (2009). No entanto, Ítavo et al. (2002) não verificaram diferenças no consumo de nutrientes digestíveis totais pela elevação da porcentagem de concentrado na dieta, atribuindo esse fato à alta quantidade de nutrientes digestíveis totais $\left(2,24 \mathrm{~kg} / \mathrm{dia}^{-1}\right)$ provenientes do volumoso. O consumo médio de energia digestível foi maior $(\mathrm{P}<0,05)$ para os animais alimentados com silagem de SDP, o que já era esperado, devido à maior quantidade de grãos na massa seca ensilada. Por outro lado, a elevação no consumo de energia digestível para os animais alimentados com a silagem de SF e a não variação no consumo dessa fração alimentar para os animais alimentados com SDP, em razão do aumento do concentrado na dieta, justifica as variações para o consumo de matéria seca.

$\mathrm{O}$ ganho de peso médio diário aumentou $(\mathrm{P}<0,05)$ com a elevação da porcentagem de concentrado para as dietas com silagem de SF, não sendo alterado nas dietas com silagem de SDP (Tab. 4), refletindo as variações no consumo de matéria seca, energia digestível e fibra em detergente neutro. Na comparação entre as diferentes silagens dos híbridos de sorgo (Tab. 4), não foi verificada diferença $(\mathrm{P}>0,05)$ no ganho de peso médio diário, resultados não esperados devido ao maior aporte energético proveniente do SDP, resultados semelhantes aos verificados por Restle et al. (2002), os quais verificaram que a silagem de sorgo duplo propósito em maior altura de corte (45 vs. $14 \mathrm{~cm})$ e maior aporte de energia não alterou o ganho de peso de bezerros Braford. Da mesma forma, Pereira et al. (2006) não verificaram alteração no ganho de peso de novilhos Holandês x Nelore alimentados com porcentagens crescentes de concentrado $(20,35,50$ e $65 \%)$ e sorgo (Volumax) como volumoso da dieta, resultados atribuídos ao elevado consumo de proteína e energia, mesmo nos menores níveis de concentrado.

A elevação do ganho de peso em virtude do aumento do concentrado nas dietas com silagem de SF foi concordante com a literatura (Missio et al., 2009; Vieira et al., 2010). Todavia, a não variação para o ganho de peso devido à elevação do concentrado na dieta para os animais alimentados com SDP não era esperada, embora sejam coerentes com a falta de variação do consumo de matéria seca. Deve-se considerar que, ao longo do experimento, os animais alimentados com silagem de SDP apresentaram biologicamente maior ganho de peso, determinando maior peso corporal e possivelmente maior exigência de mantença que aqueles alimentados com SF. Além disso, maiores ganhos de peso implicam maior taxa de deposição de gordura NRC National... (1996), o que, segundo Berg e Butterfield (1976), diminui o ímpeto de crescimento.

$\mathrm{O}$ aumento do concentrado na dieta alterou $(\mathrm{P}<0,05)$ a conversão alimentar dos novilhos alimentados com silagem de $\mathrm{SF}$, diminuindo, em média, $0,0862 \mathrm{~kg}$ de matéria seca $\mathrm{kg}^{-1}$ de ganho de peso a cada $1 \%$ a mais de concentrado na dieta (Tab. 4). Estes resultados estão associados à maior concentração energética por unidade de peso do alimento consumido, necessitando menores quantidades para atendimento das exigências de ganho de peso, em relação às dietas com menor teor de concentrado. Por outro lado, o aumento da porcentagem de concentrado na dieta não alterou significativamente a conversão alimentar dos animais alimentados com silagem de SDP, resultado da semelhança do consumo de matéria seca e do ganho de peso médio. 
Tabela 4. Desempenho de novilhos de corte em função do híbrido de sorgo e da porcentagem de concentrado na dieta

\begin{tabular}{|c|c|c|c|c|c|c|c|}
\hline \multirow{2}{*}{ Híbrido } & \multicolumn{3}{|c|}{ Concentrado $(\%)$} & \multirow{2}{*}{ Média } & \multirow{2}{*}{ Regressão } & \multirow{2}{*}{$\mathrm{R}^{2}$} & \multirow{2}{*}{$\mathrm{CV}$} \\
\hline & 25 & 35 & 45 & & & & \\
\hline \multicolumn{8}{|c|}{ Ganho de peso médio diário, $\mathrm{kg} / \mathrm{dia}^{-1}$} \\
\hline $\mathrm{SF}$ & $1,00 \mathrm{~A}$ & $1,04 \mathrm{~A}$ & $1,40 \mathrm{~A}$ & $1,15 \mathrm{~A}$ & $Y=0,454+0,0198 X$ & 0,71 & \multirow{2}{*}{10,72} \\
\hline SDP & $1,15 \mathrm{~A}$ & $1,20 \mathrm{~A}$ & $1,26 \mathrm{~A}$ & $1,21 \mathrm{~A}$ & $\mathrm{Y}=0,454$ & -- & \\
\hline \multicolumn{8}{|c|}{ Conversão alimentar, $\mathrm{kg}$ de matéria seca $\mathrm{kg}-1$ de ganho de peso corporal } \\
\hline $\mathrm{SF}$ & $9,20 \mathrm{~A}$ & $8,83 \mathrm{~A}$ & $7,47 \mathrm{~A}$ & $7,45 \mathrm{~A}$ & $\mathrm{Y}=11,515-0,0862 \mathrm{X}$ & 0,77 & \multirow{2}{*}{10,03} \\
\hline SDP & $8,20 \mathrm{~A}$ & $8,42 \mathrm{~A}$ & $7,83 \mathrm{~A}$ & $8,36 \mathrm{~B}$ & $\mathrm{Y}=8,156$ & -- & \\
\hline \multicolumn{8}{|c|}{ Dias de confinamento } \\
\hline $\mathrm{SF}$ & $96,00 \mathrm{~A}$ & $96,00 \mathrm{~A}$ & $84,00 \mathrm{~A}$ & $92,17 \mathrm{~A}$ & $\mathrm{Y}=112,293-0,575 \mathrm{X}$ & 0,69 & \multirow{2}{*}{2,98} \\
\hline SDP & $96,00 \mathrm{~A}$ & $84,00 \mathrm{~B}$ & $84,00 \mathrm{~A}$ & $88,33 \mathrm{~B}$ & $Y=108,458-0,575 X$ & 0,65 & \\
\hline
\end{tabular}

SF e SDP = sorgo forrageiro e sorgo duplo propósito, respectivamente. Médias seguidas de letras maiúsculas distintas na linha diferem entre si pelo teste Tukey a $5 \%$ de probabilidade; $\mathrm{X}=$ porcentagem de concentrado na dieta.

O número de dias em confinamento diminuiu linearmente em virtude do aumento do concentrado na dieta, diminuindo, em média, 0,575 dia a cada $1 \%$ a mais de concentrado na dieta, em ambas as silagens (Tab. 4). Os animais alimentados com silagem de SDP permaneceram menos tempo $(\mathrm{P}<0,05)$ em confinamento que os novilhos alimentados com silagem de $\mathrm{SF}$, indicando possibilidade de maior margem de lucro com a utilização de silagens de melhor qualidade. Missio et al. (2009) verificaram que a diminuição do número de dias em confinamento foi altamente correlacionada $(r=0,95)$ com a lucratividade da terminação de tourinhos superjovens. Pereira et al. (2006) concluíram que as dietas com silagem de sorgo de elevada qualidade e $20 \%$ de concentrado para bovinos machos castrados Holandês x Zebu com $435 \mathrm{~kg}$ e idade acima de 36 meses e castrados proporcionaram produção de carne com menor custo que aquelas com 35,50 e $65 \%$ de concentrado.

\section{CONCLUSÕES}

$\mathrm{O}$ aumento da porcentagem de concentrado de 25 até $45 \%$ melhora a eficiência biológica de novilhos alimentados em confinamento com silagem de sorgo forrageiro e não beneficia o sistema de alimentação com silagem de sorgo duplo propósito. Podem-se utilizar porcentagens de concentrado mais baixas na dieta de novilhos de corte quando o volumoso apresenta melhor qualidade.

\section{REFERÊNCIAS}

ANUÁRIO da Pecuária Brasileira. São Paulo: Instituto FNP, 2010. 368p.

ARAÚJO, G.G.L.; COELHO DA SILVA, J.F.; VALADARES FILHO, S.C. et al. Consumo e digestibilidade total dos nutrientes de dietas contendo diferentes níveis de volumoso, em bezerros. Rev. Bras. Zootec., v.27, p.345-354, 1998.

BERG, R.T.; BUTTERFIELD, R.M. New concepts of cattle growth. Sydney: Sydney University, 1976. 240p.

BÜRGER, P.J.; PEREIRA, J.C.; COELHO DA SILVA, J.F. et al. Consumo e digestibilidade aparente total e parcial em bezerros holandeses alimentados com dietas contendo diferentes níveis de concentrado. Rev. Bras. Zootec., v.29, p.206-214, 2000.

CHIZZOTTI, F.H.M.; PEREIRA, O.G.; VALADARES FILHO, S.C. et al. Intake, digestibility, and performance of steers fed diets based on two corn silage hybrids and two concentrate levels. Arq. Bras. Med. Vet. Zootec., v.62, p.868-874, 2010. Disponível em: $\quad<$ http://www.scielo.br/scielo.php?pid=S010209352010000400016\&script=sci_arttext $>$. Acesso em: 18 abr. 2011.

ÍTAVO, L.C.V.; VALADARES FILHO, S.C.; SILVA, F.F. et al. Consumo e digestibilidades aparentes totais e parciais de nutrientes em novilhos alimentados com dietas contendo vários níveis de concentrado. Rev. Bras. Zootec., v.31, p.1543-1552, $2002 . \quad$ Disponível em: <http://www.scielo.br/pdf/rbz/v31n3s0/13111.pdf> Acesso em: 18 abr. 2011.

MERTENS, D.R. Regulation of forage intake. In: FAHEY JR., G.C. (Ed.). Forage quality, evaluation and utilization. Madison: American Society of Agronomy. 1994. p.450-493. 
MISSIO, R.L.; BRONDANI, I.L.; FREITAS, L.S. et $a l$. Desempenho e avaliação econômica da terminação de tourinhos em confinamento alimentados com diferentes níveis de concentrado na dieta. Rev. Bras. Zootec., v.38, p.1309-1316, 2009. Disponível em: $<\mathrm{http} / / / \mathrm{www}$.scielo.br/scielo.php?script=sci_arttext\&p $\mathrm{id}=$ S1516-35982009000700021 $>$. Acesso em: $15 \mathrm{abr}$. 2011.

NEUMANN, M.; RESTLE, J.; ALVES FILHO, D.C. et al. Avaliação da silagem de diferentes híbridos de sorgo (Sorghum bicolor, L. Moench) por meio do desempenho de novilhos de corte confinados. Rev. Bras. Zootec., v.30, p.2099-2109, 2001. Disponível em: <www.scielo.br/pdf/rbz/v30n6s0/7424.pdf>. Acesso em: 18 abr. 2011.

NUTRIENTS requirements of beef cattle-NRC. 7.ed. Washington, DC: 1996. 242p.

OFFICIAL methods of analysis-AOAC. 12.ed. Washington, D.C., 1995. 1094p.

PACHECO, P.S.; RESTLE, J.; VAZ, F.N. et al. Avaliação econômica em confinamento de novilhos jovens e superjovens de diferentes grupos genéticos. Rev. Bras. Zootec., v.35, p.309-320, 2006. Disponível em: $\quad<$ http://www.scielo.br/scielo.php?pid=S151635982006000100039\&script=sci_arttext $>$. Acesso em: 15 abr. 2011.

PEREIRA, D.H.; PEREIRA, O.G.; VALADARES FILHO, S.C. et al. Consumo, digestibilidade dos nutrientes e desempenho de bovinos de corte recebendo silagem de sorgo (Sorghum bicolor (L.) Moench) e diferentes proporções de concentrado. Rev. Bras. Zootec., v.35, p.282-291, 2006. Disponível em: <http://www.scielo.br/pdf/rbz/v35n1/28371.pdf >.

Acesso em: 18 abr. 2011.

RESTLE, J.; NEUMANN, M.; BRONDANI, I.L. et al. Manipulação do corte do sorgo (Sorghum bicolor, L. Moench) para confecção de silagem, visando a produção do novilho superprecoce. Rev. Bras. Zootec., v.31, p.1481-1490, 2002. Disponível em: <http://www.scielo.br/pdf/rbz/v31n3s0/13105.pdf>. Acesso em: 18 abr. 2011.

RESTLE, J.; PACHECO, P.S.; COSTA, E.C. et al. Apreciação econômica da terminação em confinamento de novilhos Red Angus superjovens abatidos com diferentes pesos. Rev. Bras. Zootec., v.36, p.978-986, 2007. Disponível em: <www.scielo.br/pdf/rbz/v36n4/30.pdf >. Acesso em: 15 abr. 2011.
ROBERTSON, J.B.; VAN SOEST, P.J. The detergent system of analysis. In: JAMES, W.P.T.; THEANDER, $\mathrm{O}$. The analysis of dietary fiber in food. New York: Marcel Dekker, 1981. p.123-158.

SILVA, B.C.; PEREIRA, O.G.; PEREIRA, D.H. et al. Consumo e digestibilidade aparente total dos nutrientes e ganho de peso de bovinos de corte alimentados com silagem de Brachiaria brizantha e concentrado em diferentes proporções. Rev. Bras. Zootec., v.34, p.1060-1069, 2005. Disponível em: <http://www.scielo.br/scielo.php?pid=S151635982005000300039\&script=sci_arttext $>$. Acesso em: 15 abr. 2011.

TIBO, G.C.; VALADARES FILHO, S.C.; VALADARES, R.F.D. et al. Níveis de concentrado em dietas de novilhos mestiços F1 Simental x Nelore. 1. Consumo e digestibilidade. Rev. Bras. Zootec., v.29, p.910-920, 2000.

TILLEY, J.M.A.; TERRY, R.A.A. Two stage technique for the in vitro digestion of forage crops. $J$. Brit. Gras. Soc., v.18, p.104-111, 1963.

NUTRIENT requirements of ruminants livestock. London: CAB International, 1980. 351p.

VAN SOEST, P.J. Nutritional ecology of the ruminant. 2nd ed. Ithaca, NY: Cornell University Press. 476p. 1994

VÉRAS, R.M.L.; VALADARES FILHO, S.C.; AZEVÊDO, J.A.G. et al. Níveis de concentrado na dieta de bovinos Nelore de três condições sexuais: consumo, digestibilidades total e parcial, produção microbiana e parâmetros ruminais. Rev. Bras. Zootec., v.37, p.951-960, 2008. Disponível em: <http://www.scielo.br/scielo.php?script=sci_arttext\&p $\mathrm{id}=\mathrm{S} 1516-35982008000500025$ >. Acesso em: 15 abr. 2011.

VIEIRA, B.R.; OBEID, J.A.; PEREIRA, O.G. et al. Consumo, digestibilidade dos nutrientes e parâmetros ruminais em bovinos alimentados com silagem de capim Mombaça. Arq. Bras. Med. Vet. Zootec., v.62, p.1148-1157, 2010. Disponível em: <http://www.scielo.br/pdf/abmvz/v62n5/18.pdf> Acesso em: 15 abr. 2011.

WILKINSON, J. Transformações e perspectivas dos agronegócios brasileiros. Rev. Bras. Zootec., v.39, p.26-34, 2010. Disponível em: $<$ http://www.scielo.br/scielo.php?pid=S1516-

35982010001300004\&script=sci_arttext> Acesso em: 15 abr. 2011. 